\title{
Variations in Bilingual Processing of Positive and Negative Information
}

\author{
Elsie Ong(a), Samara Hussain(b), Yvonne Chow (b), and Catherine Thompson(a)

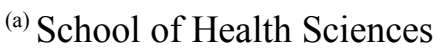 \\ University of Salford \\ (b) $\mathrm{LiPACE}$ \\ OUHK \\ Salford, UK \\ Kwai Chung, Hong Kong
}

\begin{abstract}
Past research suggests that the emotional content of words has greater impact when presented in a bilingual's first language (L1) compared to their second language $(\mathrm{L} 2)$. This is predicted to be a consequence of automatic processing of emotional words in L1 compared to slower, semantic processing in L2. In the current study 58 Chinese-English bilinguals from Hong Kong rated the valence and arousal of positive, neutral, and negative words presented in Chinese (L1) and English (L2). In contrast to predictions, perceived emotionality of the words was higher in L2, with positive words rated more positively and negative words rated more negatively when presented in English compared to Chinese. The findings suggest that words presented in $\mathbf{L} 2$ did not have lower emotional impact than L1, the results indicate that emotional processing of words may be influenced by language proficiency and language complexity.
\end{abstract}

Keywords- valence, arousal, language processing, bilinguals, emotion.

\section{INTRODUCTION}

Words are the building blocks of language and they are fundamental in providing meanings that can be understood and shared between people [1]. The semantic processing of words begins as early as infancy and this skill is so well mastered that children can quickly understand thousands of new words flexibly and creatively to allow for effective communication. According to [1], word comprehension is based on the mental lexicon; a store of word-specific information that is associated with semantic knowledge of each word. Emotional words seem to hold a special significance in the mental lexicon because both the language system and the emotional regulation system develop together during childhood. This means that words that are learned early are more tightly connected to the brain's emotional system [2]. This also indicates that words carry both a semantic meaning and an emotional meaning via amygdala mediated learning. Based on this, early theoretical frameworks have posited that language and emotion are highly interlinked [3][4][5]. For example, research has shown that language can influence the intensity of emotions (including anger, disgust, fear, and sadness) being experienced and perceived [6]. Work also reveals that diminishing one's access to the meaning of emotional words also diminishes the ability to perceive the emotional content of stimuli [7]. These findings suggest that access to the meaning of emotional words is an important element for recognizing emotional information [6], and that language processing has an impact on the ability to detect emotion.

\section{A. Emotional word processing versus neutral word processing}

Aside from the ongoing investigations of how emotion and language are intertwined, it has long been established that a processing advantage is evident for emotional information, with emotional stimuli being processed quicker than neutral stimuli [8][9][10]. To illustrate this, lexical decision tasks have been used as measures of implicit emotional word processing. Participants are required to categorize words as real words or non-words and responses are quicker to emotional words than neutral words [11] (see [12] for a discussion). This phenomenon can be explained using the motivational account of information processing [13]. It is posited that emotional information is generally more relevant for survival and wellbeing and consequently there is greater motivation to prioritize emotional stimuli. In addition, the high activation of the emotional system in the brain during the processing of emotional words (and other stimuli) means that emotional words recruit more attention and elicit an earlier and deeper level of processing than neutral words.

The processing advantage for emotional words compared to neutral words has also been found using other cognitive tasks such as the Emotional Stroop task [14][15]. This task provides an experimental measure of selective attention to emotional information. Participants are presented with emotional and neutral words in different colours and are asked to respond to the colour of each word. Results show slower responses to the colour of negative words (e.g. death) compared to positive (e.g. love) and neutral (e.g. work) words [16][17][18], and this is referred to as the Emotional Stroop effect [19][16]. As reading is an automatic process a participant must inhibit the word itself in the Stroop task and focus on the colour. The Emotional Stroop effect shows that emotional words (particularly negative words) capture attention more readily than other words and are more difficult to inhibit (e.g., [20][15]; see [21] for a review). Again, this shows that emotional stimuli have a special significance on cognitive processing.

\section{B. Language processing in monolinguals and bilinguals}

Despite robust findings regarding the link between emotion and language, the majority of investigations using words as stimuli have been carried out using monolingual participants (especially English speakers) rather than bilinguals. A bilingual speaker is defined as someone with the ability to use two languages with nearly equal fluency [22][23]. Given the 
assumption that processing of the second language (L2) is generally less efficient and automatic than processing of the first language (L1) in bilingual speakers (see [24], for a review). This raises questions as to whether the interaction between emotion and language processing is replicable within a bilingual population. One way to explore this is to investigate the difference in intensity of emotions experienced and expressed (also referred to as emotionality) in L1 compared to L2. Past findings indicate that L2 has less emotional impact relative to L1 in bilinguals [25][26] and this is attributed to the fact that emotional words learned in L1 are coded more deeply because they are experienced in a wider range of contexts and are applied in more situations compared to the words learned in L2 [18]. Therefore, the emotional impact for L1 emotion representations is stronger than for L2 emotion representations. Consistent with this view, bilingual speakers are generally more willing to discuss potentially uncomfortable topics (e.g. sex) in their L2 than their L1 [27][28][29][30].

To investigate the effect of bilingualism on emotional processing [31] conducted a study using a rapid serial visual presentation (RSVP) task comparing English monolinguals and Chinese-English bilinguals. Participants were asked to respond to targets presented in a stream containing distractor words (neutral and taboo/sexual words presented in English). Results showed that the taboo words were more distracting, but only for English monolinguals. Chinese bilinguals were less distracted by the taboo words and were better able to ignore the emotional distractors. The researchers argue that this is because emotionality is reduced in L2. Consistent with this, bilinguals often report that although they know the emotional meaning of words in L2, the words do not trigger the same affect as they do when presented in L1 [26]. It has been proposed that the dissociation between processing of emotional information in L1 and L2 is because bilinguals can process information presented in their L2 semantically but not affectively [32]. This means that although bilinguals understand the affective meaning of emotional words presented in L2, the words are unable to trigger the heightened level of automatic arousal that occurs in L1. In support of this explanation, bilinguals generally have greater autonomic and psychophysiological activation for $\mathrm{L} 1$ processing relative to $\mathrm{L} 2$ [33][34]. It has therefore been suggested that whilst L1 processing relies on rapid and automatic activation of semantic and affective information simultaneously, L2 processing is slower and parallel activation of semantic and emotional information is not possible.

More recently, the difference between emotional processing in L1 and L2 has also been investigated by measuring activation in the brain. For instance, the dissociated pattern of neurological activations elicited by L1 versus L2 using event related potentials is one typical method to investigate the automatic sub-processes in emotional word processing [35]. The early posterior negativity (EPN) gives an indirect measure to show which emotional words capture attention for sustained processing and activation of the emotion regulation system [36]. Findings illustrate that emotional words of both L1 and L2 have enhanced EPN relative to neutral words. More importantly, L1 and L2 do not differ in the amplitude of the EPN and results show similar processing of emotion words in
L1 and L2. The main difference between L1 and L2 is that words presented in L2 elicit longer delays of EPN compared to when presented in L1. This implies that processing of emotionality of L2 words is slower than L1 as a result of delayed lexical access. Although emotionality of both languages may be equivalent, as shown by comparable amplitude in EPN and similar emotionality of words, the delayed processing of L2 is often attributed to L2 having lower emotionality than L1 when in fact it is due to the different processing of L1 and L2 [35].

\section{Early versus late bilinguals}

Currently, more than half the world's population is bilingual and each culture has its own emotional lexicon for the classification of experiences [37][38]. The way bilinguals deal with their two languages has long been the topic of psychological research and the main focus has been the simultaneous activation of the two language systems (L1 and L2), or the cognitive costs of switching between L1 and L2 for ERP studies [39][40][30][41]. As stated earlier, the emotional content of words is less available when presented in L2 compared to L1, and findings show that this difference is more pronounced when L2 is learned later in life, showing a dissociated emotional effect between late bilinguals and early bilinguals [16]. Importantly, if bilinguals have learnt their L2 early in life (e.g. during infancy), then both L1 and L2 are equally capable of activating the emotional response triggered by the word stimuli. This assumption was confirmed by [34] using physiological correlates and found that skin conductance response of bilinguals to emotional words in L2 was negatively correlated with the age of L2 acquisition. It is noted that in early bilinguals (L2 learnt prior to 7 years of age), the processing of emotionality is the same in both L1 and L2, whereas in late sequential bilinguals, L2 is associated with lesser emotional impact. According to [30], this difference is due to the fact that in late-onset bilinguals L2 is typically learned in formal contexts such as school, university, or the workplace and as a consequence the link between affective processing is missing, compared to language learnt in childhood. Therefore, the emotional valence of L2 words is considered "disembodied" and the emotionality of information in L2 is processed less effectively than in L1 [32].

Consistent with this finding, [34] found that while late bilinguals showed stronger skin conductance responses when reading emotional phrases in $\mathrm{L} 1$ relative to $\mathrm{L} 2$, early bilinguals showed no difference. Together with the work that measures brain activity when bilinguals respond to emotional words, this shows that although there may be a difference in the cognitive processing of L1 and L2 for bilinguals semantically, there is no difference in the arousal response being triggered neurologically or physiologically.

\section{Language proficiency and emotional processing}

Despite the supporting evidence for decreased L2 emotionality in bilingual speakers, some researchers suggest that language proficiency may play a major role in the interaction between emotion and language. Using an 
Emotional Stroop task, [17] and [18] both investigated emotional processing in bilinguals. The study by [17] used late-onset bilinguals and the study conducted by [18] used early-onset bilinguals. Both found evidence of the Emotional Stroop effect whereby the colour of negative words was identified slower than the colour of neutral words. Crucially this effect was apparent in both L1 and L2 across both studies. This contrasts with earlier research showing that age of onset influences the processing of emotional words. Based on these findings [17] suggest that level of proficiency might be the critical factor in explaining the dissociation in processing between L1 and L2, rather than the age of acquisition. The conclusion drawn from these studies outlined that for proficient bilinguals, emotional words in L2 are processed automatically in the same way as words in L1. Proficient bilinguals can elicit strong and automatic associations between emotion and meaning in L2 and therefore show the same emotionality in L1 and L2.

A further important factor that may influence emotional language processing of bilinguals is the language itself. A study by [17] measured processing of Finnish-English bilinguals and a study by [18] measured processing of Spanish-English bilinguals and both found consistent effects. However a more recent study by [42] did not support these findings. Highly proficient Turkish-English bilinguals were asked to make responses to emotional words presented in L1 and L2 (requiring processing at a shallow or deep level). They were given a surprise recall test and recall was better for emotional L2 words than L1 words. Participants also reported increased sensitivity to emotional L2 words compared to L1 words. The researchers proposed that the English language elicits strong emotionality compared to Turkish, therefore making the case that the characteristics of L1 and L2 may play a role in the emotional processing of language by bilinguals [42]. This suggests that by investigating emotional processing in monolingual English speakers, it is impossible to fully explore and understand human responses to emotional stimuli.

\section{E. Aims and intentions of the current experiment}

The aim of this study is to explore how bilinguals respond to emotional words presented in L1 and L2. All participants will be of a high proficiency and are Chinese-English bilinguals. Although Chinese is the world's oldest written language [43] and the most popular spoken language in the world [44], very few research studies have explored whether there are differences in how Chinese-English bilinguals process emotional information in their first and second language. In addition to measuring the processing of emotionality in words for bilinguals, this study will also provide information about how the characteristics of the language may play a role in emotionality. Chinese is very distinctive and the difference between Chinese and English is much greater than the differences between English and languages such as Spanish, Dutch, and French [45][5].
The work will specifically address how an individual assesses the emotionality of words in L1 and L2 by asking participants to rate a series of positive, negative, and neutral words. Research measuring emotional processing tends to use stimuli that have been manipulated according to two measures; valence and arousal [46]. Valence refers to how negative or positive a stimulus is, and arousal refers to how calm or excited the stimulus makes the individual feel [47][13]. The current study will make use of the Affective Norms for English Words (ANEW; [47]), a database of approximately 1000 words that have each been rated for valence and arousal. The ANEW lists have been translated into other languages including Dutch [48], Finnish [4], German [49], Italian [50], Portuguese [51], and Spanish [52]. However, the ANEW has not been translated into Chinese and there is little knowledge of how the valence and arousal of English words will compare with the Chinese translation.

The current study will therefore compare self-rated emotional valence and arousal of L1 (Chinese) and L2 (English) words for Chinese-English bilinguals. By comparing words in L1 versus L2, the study can explore how the interaction of language and emotion may be processed in bilinguals. It is predicted that ratings of valence and arousal will be significantly different in L1 and L2 of bilingual speakers. Method

\section{F. Design}

This study compared valence and arousal ratings of words presented in Chinese and English to Chinese-English bilinguals. A $2 \times 3$ within-participants design was used with two independent variables; the language each word was presented in: Chinese (L1) or English (L2), and the emotion of words: positive, negative, or neutral. The dependent variables were the mean ratings of arousal and valence.

\section{G. Participants}

Participants were an opportunity sample of 58 undergraduate Psychology students (40 females, 18 males) recruited from The Open University Hong Kong. Their ages ranged from 18 to 52 years, with a mean age of 23.17 years $(\mathrm{SD}=6.61)$. All participants were Chinese-English bilinguals. They were all studying for a degree in their L2 so were considered proficient in both languages, and all acquired L2 prior to the age of 7 .

\section{H. Materials}

One hundred and twenty English words (40 positive, 40 neutral, and 40 negative) were selected from the ANEW [47]. Each word had been rated for valence and arousal on a 9-point scale, with 1 being lowest, and 9 being highest. The positive words had a mean valence of 7.52 (ranging from 7.06 to 8.33) and a mean arousal of 6.90 (ranging from 6.74 to 7.83 ). The negative words had a mean valence of 2.32 (ranging from 1.29 to 2.93 ) and a mean arousal of 6.72 (ranging from 6.33 to 7.86). The neutral words had a mean valence of 5.42 (ranging from 5.06 to 6.26) and a mean arousal of 3.82 (ranging from 3.22 to 4.34). All words were obtained from the list in English and translated into Chinese using two dictionary sources 
[22][23]. Participants rated the valence and arousal of each word using the 9-point Self-Assessment Manikin scale (SAM; [53]. The valence scale consisted of 9 faces ranging from an unhappy, frowning face (1) to a smiling, happy face (9). The arousal scale consisted of 9 faces ranging from a relaxed, sleepy face (1) to an excited, wide-eyed face (9).

\section{Procedure}

The words were presented in a pseudo-random order on a sheet of paper and participants were asked to rate the arousal and valence of each word. All participants saw each word once therefore for each participant half the words (20 negative, 20 neutral, and 20 positive) were presented in English and the other half were presented in Chinese. The language of presentation and the order of presentation were counterbalanced across participants. Participants were given six practice words and if they were satisfied with the procedure they continued. They were asked to complete the task as quickly as possible and overall the ratings took a maximum of 20 minutes to complete.

\section{RESULTS}

Mean ratings for valence and arousal were calculated for each category of emotion for each language. The effect of language and emotion were analyzed using two 2 (language) $\mathrm{x}$ 3 (emotion) within-participant ANOVAs (one for valence and one for arousal). Significant effects were further investigated using t-tests with Bonferroni corrections. Where sphericity was violated, corrected degrees of freedom are reported using the greenhouse geisser.

For the analysis of valence (see table 1 for valence ratings), there was a significant effect of emotion, $F(1.341,76.425)=$ 121.572, $\mathrm{MSE}=3.250, \mathrm{p}<.001$. Pairwise comparisons indicated that participants rated the valence of positive words higher valence the neutral words, $\mathrm{t}(57)=17.520, \mathrm{p}<.001$, and rated the valence of negative words significantly lower than the neutral words, $\mathrm{t}(57)=-4.816, \mathrm{p}<.001$.

TABLE I. MEAN VALENCE RATINGS FOR EACH CATEGORY OF WORD WHEN PRESENTED IN CHINESE AND IN ENGLISH

\begin{tabular}{|l|l|l|l|l|}
\hline Emotion & Negative & Neutral & Positive \\
\hline \multirow{4}{*}{ Language } & Chinese & 4.18 & 4.20 & 6.22 \\
& & $(2.38)$ & $(1.45)$ & $(1.44)$ \\
\cline { 2 - 5 } & \multirow{2}{*}{ English } & 2.93 & 4.89 & 6.82 \\
& & $(0.86)$ & $(0.44)$ & $(0.84)$ \\
\hline & Mean & 3.55 & 4.54 & 6.52 \\
& & $(1.89)$ & $(1.12)$ & $(1.21)$ \\
\hline
\end{tabular}

There was no significant effect of language, $\mathrm{F}(1,57)=$ $0.022, \mathrm{MSE}=1.203, \mathrm{p}>.05$. However, there was a significant interaction between language and emotion, $\mathrm{F}(1.170,66.668)=$ $15.725, \mathrm{MSE}=3.772, \mathrm{p}<.001$. Pairwise comparisons showed that participants rated negative words as significantly more negative when presented in English, $\mathrm{t}(57)=-3.729, \mathrm{p}<.001$, compared to when presented in Chinese. They also rated positive words as more positive when presented in English compared to Chinese, $\mathrm{t}(57)=3.329, \mathrm{p}<.005$. For neutral words, the Chinese words were rated as having a lower mean valence than the English words, $\mathrm{t}(57)=3.120, \mathrm{p}<.005$.
For the analysis of arousal (see table 2 for arousal ratings), there was a significant effect of emotion, $\mathrm{F}(1.353,77.142)=$ $37.811, \mathrm{MSE}=2.075, \mathrm{p}<.001$. Pairwise comparisons revealed that participants rated arousal of positive words, $\mathrm{t}(57)=$ $12.269, \mathrm{p}<.001$, and negative words, $\mathrm{t}(57)=4.951, \mathrm{p}<.001$ higher than the neutral words.

TABLE II. MEAN AROUSAL RATINGS FOR EACH CATEGORY OF WORD WHEN PRESENTED IN CHINESE AND IN ENGLISH

\begin{tabular}{|l|l|l|l|l|}
\hline Emotion & Negative & Neutral & Positive \\
\hline \multirow{4}{*}{ Language } & Chinese & 5.34 & 2.64 & 5.00 \\
& & $(1.80)$ & $(1.59)$ & $(1.62)$ \\
\cline { 2 - 5 } & \multirow{2}{*}{ English } & 4.04 & 3.88 & 5.77 \\
& & $(1.69)$ & $(1.73)$ & $(1.69)$ \\
\hline & Mean & 4.69 & 3.26 & 5.39 \\
& & $(1.85)$ & $(1.77)$ & $(1.69)$ \\
\hline
\end{tabular}

There was no significant effect of language, $\mathrm{F}(1,57)=$ $2.327, \operatorname{MSE}=2.137, \mathrm{p}>.05$. However again there was a significant interaction between language and emotion, $F$ $(1.353,77.142)=37.811, \mathrm{MSE}=2.075, \mathrm{p}<.001$. Pairwise comparisons between the two languages illustrated that participants rated negative words as more arousing when presented in Chinese, $\mathrm{t}(57)=-5.361, \mathrm{p}<.001)$. They also rated positive words as more arousing when presented in English, $t$ $(57)=3.183, p<.005$. Neutral words presented in Chinese were rated as having a lower arousal than neutral words presented in English, t (57) $=5.428, \mathrm{p}<.001$.

\section{DISCUSSION}

The aim of the current study was to investigate emotional responses of Chinese-English bilinguals to emotional words presented in their first and second language. This was achieved by asking participants to rate the valence and arousal of positive, negative, and neutral words shown in Chinese (L1) and English (L2). The majority of past findings show a processing advantage in L1 whereby emotional words in L1 are processed automatically at both a semantic and emotional level whilst emotional words in L2 are processed for meaning before emotionality [25][26]. It was predicted that words shown in Chinese would elicit higher emotionality than words shown in English, however most work in this area compares bilingual processing across similar languages such as English and French and very little work has been conducted using Chinese. Given that [42] have argued that the characteristics of a language may affect emotionality the current study had the potential to show whether this may be a factor in emotional processing.

In contrast to the predictions made participants rated positive English words with higher valence than the Chinese translated words and negative English words with lower valence than the Chinese translations. Participants rated the arousal of negative Chinese words higher than negative English words but this pattern was reversed for positive words, with arousal ratings higher for positive English words than Chinese words. These findings show that language does influence emotional processing, L1 does not necessarily hold a processing advantage, and the nature of the language may influence emotionality. 
So far studies conducted on emotional language processing of bilinguals have yielded conflicting results. Some show processing advantages for L1 [54], others provide evidence for an advantage in L2 [55][42], and some show no difference between L1 and L2 [42][56][57][18]. The discrepancies between these findings may indicate that L2 processing is not always less emotional than L1 but is dependent on a variety of factors such as age of second language acquisition [27][16], and language proficiency [42][17]; see also [18]. The current findings also add to the argument that the characteristics of a language may have an impact.

The participants in this study were all Chinese-English bilinguals currently residing in Hong Kong having also been educated in Hong Kong. They all fall into the category of 'early bilinguals' as English (L2) was acquired prior the age of seven [58]. According to the Pre-primary Curriculum issued by the Hong Kong Education Bureau in 2006, the learning objectives for children in Hong Kong aged 2-6 were to develop an interest in learning English along with Chinese. The curriculum in English development includes listening and reading English stories and nursery rhymes, recognizing and making use of English vocabulary in everyday life, and understanding simple English conversation [59][58]. Bilinguals in the current study grew up with this curriculum and have learnt English as early as Kindergarten at aged 3.

This is consistent with a neurological finding by [45] using Chinese English early-onset bilinguals that also showed comparable understanding of the emotional meaning of words presented in L1 and L2. Notably, although L1 word processing has gained advantage in relying on the rapid and automatic attention capture, L2 word processing is compensated by facilitation via semantic retrieval which might help elicit comparable understanding of words in L2. In the current study, Chinese-English bilinguals rated their L2 as having higher emotionality than their L1 showing that they have high semantic retrieval of the English words. The results also reflect that both Chinese and English may be closely linked to the emotional content of the lexicon associated with each language. In contrast, if the participants were late sequential bilinguals where L2 is learned in the teenage years during school, university or in adulthood workplace, L2 may be more distantly associated with the underlying emotions and will be considered as less arousing than L1 [27][16]. Consequently, "the same opportunities for affective linguistic conditioning" are not present in comparison with the L1 learning in early childhood ([30], p.156). It is argued that when individuals learn a language early in life they learn to associate it with different situations and contexts and so the emotional content of the language becomes much more apparent. This is different to learning a language in later life as emotion is not so closely associated with language. As the present study used early-onset bilinguals this may explain why L1 emotional words were not rated as more emotional.

Regarding the comparisons of arousal ratings in L1 and L2, participants rated negative words in Chinese (L1) as more arousing than in L2 and positive words in English (L2) as more arousing than when shown in L1. Therefore results were inconsistent with predictions and past literature that bilinguals report more emotional distance with words presented in L2 relative to L1 [27][19][30]. The disparity between these findings can again be explained by the English proficiency levels of the Chinese-English bilinguals participants in the current study, and the context in which these participants have learned English. Past studies have shown that activation of emotions in the two languages and the extent of arousal elicited from emotional word processing are dependent on language proficiency and how the language was acquired [33][34][32]. Related to this, the current model of organization of the bilingual lexicon suggests that the mental lexicon in L2 users becomes more integrated across the L1 and L2 as proficiency develops [60]. For early bilinguals with high L2 proficiency, a word presented in one language automatically activates, in parallel, the mental representation of the corresponding translation in the other language.

In the current study, although participants may consider Chinese as their L1 and English as L2, their frequent use of English for studying their full-time overseas degree program should be accounted for. These students may have high proficiency for L2 in addition to high frequency of L2 usage. Both may contribute to higher emotionality of L2. This is consistent with the arguments of [61] who have explored automatic emotional language processing in bilinguals with similar characteristics as the participants in the present study. The study recruited French German bilinguals who were not immersed in an L2 culture but used L2 frequently in daily life. Their results confirmed semantic processing effects and emotionality in both L1 and L2 with comparable degrees of spontaneous and fast word processing regardless on language. This indicates frequency of use is an important factor together with age of acquisition and proficiency of L2 [61][35]. Indeed, processing of L2 can elicit high emotionality just like L1 if L2 is used frequently and was learned via immersion rather than in the classroom [29][61]. If language is being learnt and habitually used in a specific emotional context, it will enable more emotional resonance as human experiences are learnt and stored in a context-dependent manner [34][3]. The present findings therefore support the conclusion of [61] that these factors are related to the level of language immersion of bilinguals (although frequency of use is arguably claimed to be the most critical factor rather than context of use). To confirm this assumption it would be worthwhile to explore the characteristics of bilinguals in more depth for future studies. A bilingual's early childhood language development, type of language, and their language usage frequency should be measured objectively to correlate with language emotionality.

\section{CONCLUSION}

The current research compared perceived emotionality (valence and arousal) of words presented in Chinese and English to Chinese-English bilinguals. In contrast to the predictions made participants rated English words as more emotional than Chinese words, therefore providing no support for the argument that emotional stimuli presented in one's first language is automatically processed at both a semantic and emotional level, whereas stimuli presented in the second language is processed at a semantic level. The current findings emphasized that the Chinese-English comparison is important and unique when compared to other languages since the results do not follow similar patterns when compared to other groups 
such as French-English or German English bilinguals. These findings support past research suggesting that age of acquisition, frequency of use, and language proficiency all influence emotionality, however they further show that the characteristics of a language may also have an impact on emotional processing.

\section{REFERENCES}

[1] Nation, K. (2013). Lexical learning and lexical processing in children with developmental language impairments. Philosophical Transactions of the Royal Society B: Biological Sciences, 369(1634), pp.2012038720120387.

[2] Bloom, L. and Beckwith, R. (1989). Talking with Feeling: Integrating Affective and Linguistic Expression in Early Language Development. Cognition \& Emotion, 3(4), pp.313-342.

[3] Caldwell-Harris, C. (2014). Emotionality differences between a native and foreign language: theoretical implications. Frontiers In Psychology, 5 .

[4] Eilola, T., \& Havelka, J. (2010). Affective norms for 210 British English and Finnish nouns. Behavior Research Methods, 42(1), 134-140.

[5] Ho, S., Mak, C., Yeung, D., Duan, W., Tang, S., Yeung, J., \& Ching, R. (2015). Emotional Valence, Arousal, and Threat Ratings of 160 Chinese Words among Adolescents. PLOS ONE, 10(7), e0132294.

[6] Lindquist, K. A., MacCormack, J. K., \& Shablack, H. (2015). The role of language in emotion: predictions from psychological constructionism. Frontiers in Psychology, 6(444), 1-17.

[7] Havas, David A. and James Matheson. "The Functional Role Of The Periphery In Emotional Language Comprehension". Frontiers in Psychology 4 (2013): n. pag. Web.

[8] Kuchinke, L., Võ, M. L.-H., Hofmann, M., \& Jacobs, A. M. (2007). Pupillary responses during lexical decisions vary with word frequency but not emotional valence. International Journal of Psychophysiology: Official Journal of the International Organization of Psychophysiology, 65(2), 132-40.

[9] Scott, G. G., O'Donnell, P.J., Leuthold, H., \& Sereno, S.C. (2009). Early emotion word processing: Evidence from event-related potentials. Biological Psychology, 80, 95-104.

[10] Kuperman, V., Estes, Z., Brysbaert, M., \& Warriner, A.B. (2014). Emotion and Language: Valence and Arousal Affect Word Recognition. Journal of Experimental Psychology. General.

[11] Kousta, S.-T., Vinson, D. P., \& Vigliocco, G. (2009). Emotion words, regardless of polarity, have a processing advantage over neutral words. Cognition, 112(3), 473-81.

[12] Vinson, D., Ponari, M., \& Vigliocco, G. (2014). How does emotional content affect lexical processing? Cognition \& Emotion, 28(4), 737-746.

[13] Lang, P. J., Bradley, M. M., \& Cuthbert, B. (1997). Motivated attention: Affect, activation, and action. In P. Lang, R. Simons, \& M. Balaban (Eds.), Attention and Orienting: Sensory and Motivational Processes (pp. 97-135). Erlbaum, Hillsdale NJ.

[14] Citron, F. M. M., Weekes, B. S., \& Ferstl, E. C. (2014). Arousal and emotional valence interact in written word recognition. Language, Cognition and Neuroscience, 29(10), 1257-1267.

[15] Gilboa-Schechtman, E., Revelle, W. and Gotlib, I. (2000). Journal search results - Cite This For Me. Cognitive Therapy and Research, 24(5), pp.491-502.

[16] Dewaele, J.-M., \& Pavlenko, A. (2002). Emotion Vocabulary in Interlanguage. Language Learning, 52(2), 263-322.

[17] Eilola, T. M., Havelka, J., \& Sharma, D. (2007). Emotional activation in the first and second language. Cognition \& Emotion, 21(5), 1064-1076.

[18] Sutton, T. M., Altarriba, J., Gianico, J. L., \& Basnight-Brown, D. M. (2007). The automatic access of emotion: Emotional Stroop effects in Spanish-English bilingual speakers. Cognition \& Emotion, 21(5), 10771090.

[19] Dewaele, J.-M. (2004). Perceived language dominance and language preference for emotional speech: The implications for attrition research. In M. Schmid (Ed.), First language attrition: Interdisciplinary perspectives on methodological issues (pp. 81-104).
[20] Pratto, F. and John, O. (1991). Automatic vigilance: The attentiongrabbing power of negative social information. Journal of Personality and Social Psychology, 61(3), pp.380-391.

[21] Larsen, R. J., Mercer, K. A., \& Balota, D. A. (2006). Lexical Characteristics of Words Used in Emotional Stroop Experiments. Emotion, 6(1), 62-72.

[22] Cambridge Dictionary | Free English Dictionary, T. (2015). Cambridge Dictionary | Free English Dictionary, Translations and Thesaurus. Dictionary.cambridge.org. Retrieved 11 November 2015, from http://dictionary.cambridge.org/

[23] Longman English -Chinese Dictionary of Contemporary English (1988). Longman Group (Far East) Limited, Hong Kong.

[24] Van Heuven, W.J.B., Mandera, P., Keuleers, E., \& Brysbaert, M. (in press). Subtlex-UK: A new and improved word frequency database for British English. Quarterly Journal of Experimental Psychology.

[25] Marian, V. and Kaushanskaya, M. (2004). Self-construal and emotion in bicultural bilinguals. Journal of Memory and Language, 51(2), pp.190201.

[26] Pavlenko, A. (2005). Emotions and Bilingualism. New York: Cambridge University Press.

[27] Altarriba, J. (2008). Expressions of emotion as mediated by context. Bilingualism: Language and Cognition, 11(02), 165-167.

[28] Altmann, U., Bohrn, I., Lubrich, O., Menninghaus, W., \& Jacobs, A. (2012). The power of emotional valence-from cognitive to affective processes in reading. Frontiers In Human Neuroscience, 6.

[29] Dewaele, J. M. (2010). Emotions in Multiple Languages. London: Palgrave Macmillan.

[30] Pavlenko, A. (2008). Emotion and emotion-laden words in the bilingual lexicon. Bilingualism: Language and Cognition, 11, 147-164.

[31] Colbeck, K. L., and Bowers, J. S. (2012). Blinded by taboo words in L1 but not L2. Emotion 12(2), 217-222.

[32] Pavlenko, A. (2012). Affective processing in bilingual speakers: disembodied cognition? International Journal of Psychology, 47(6), 405-428.

[33] Harris, C. L., Ayçiçegi, A., \& Gleason, J. B. (2003). Taboo words and reprimands elicit greater autonomic reactivity in a first language than in a second language. Applied Psycholinguistics, 24, 561-579.

[34] Harris, C. L., Gleason, J. B., and Ayçiçegi, A. (2006). "When is a first language more emotional? Psychophysiological evidence from bilingual speakers," in Bilingual Minds: Emotional Experience, Expression, and Representation. Clevedon, ed A. Pavlenko (Clevedon: Multilingual Matters), 257-283.

[35] Opitz, B., \& Degner, J. (2012). Emotionality in a second language: It's a matter of time. Neuropsychologia, 50(8), 1961-1967.

[36] Schacht. ,A.,and Sommer, W.(2009). Emotions in word and face processing: early and late cortical responses. Brain Cognition. 69, 538550.

[37] Sianipar, A., Middelburg, R., \& Dijkstra, T. (2015). When feelings arise with meanings: How emotion and meaning of a native language affect second language processing in adult learners. PLoS ONE, 10(12), e0144576.

[38] Yik, M. (2010). How unique is Chinese emotion, in The Oxford Handbook of Chinese Psychology. (eds. Bond, M. H.). Oxford University Press: USA.

[39] Chauncey K, Grainger J, Holcomb PJ. Code-switching effects in bilingual word recognition: A masked priming study with ERPs. Brain and Language. 2008;105:161-174.

[40] Midgley, K. J., Holcomb, P. J., Van Heuven, W. J., \& Grainger, J. (2008). An electrophysiological investigation of cross-language effects of orthographic neighborhood. Brain Res, 1246, 123-135.

[41] Van Der Meij, M., Cuetos, F., Carreiras, M., \& Barber, H. A. (2011). Electrophysiological correlates of language switching in second language learners. Psychophysiology, 48(1), 44-54.

[42] Ayçiçegi-Dinn, A., \& Caldwell-Harris, C. L. (2009). Emotion-memory effects in bilingual speakers: A levels-of-processing approach. Bilingualism: Language and Cognition, 12(03), 291. 
[43] Oxford Dictionaires (10 April 2015). Infographic: Chinese language facts. Retrieved

http://blog.oxforddictionaries.com/2015/04/infographic-chineselanguage-facts/

[44] Ethnologue (2014). Languages of the World. Retrieved from http://www.ethnologue.com/world

[45] Chen, P., Lin, J., Chen, B., Lu, C., \& Guo, T. (2015). Processing emotional words in two languages with one brain: ERP and fMRI evidence from Chinese-English bilinguals. Cortex, 71, 34-48.

[46] Warriner, A. B., Kuperman, V., \& Brysbaert, M. (n. d.). Norms of valence, arousal, and dominance for 13,915 English lemmas. Retrieved from

http://www.uvm.edu/ pdodds/research/papers/files/2013/warriner2013a. pdf

[47] Bradley, M M., \& Lang, P J. (1999). Affective norms for English words (ANEW): Stimuli, instruction manual and affective ratings. Technical report C-1. Gainesvill, FL: The Center for Research in Psychophysiology, University of Florid.

[48] Moors, A., De Houwer, J., Hermans, D., Wanmaker, S., van Schie, K., \& Van Harmelen, A. et al. (2012). Norms of valence, arousal, dominance, and age of acquisition for 4,300 Dutch words. Behaviour Research Methods, 45(1), 169-177.

[49] Kanske, P., \& Kotz, S. (2011). Cross-modal validation of the Leipzig Affective Norms for German (LANG). Behavior Research Methods, 43(2), 409-413.

[50] Montefinese, M., Ambrosini, E., Fairfield, B., \& Mammarella, N. (2014). The adaptation of the Affective Norms for English Words (ANEW) for Italian. Behaviour Research Methods, 46(3), 887-903.

[51] Soares, A., Comesaña, M., Pinheiro, A., Simões, A., \& Frade, C. (2011). The adaptation of the Affective Norms for English Words (ANEW) for European Portuguese. Behaviour Research Methods, 44(1), 256-269.
[52] Ferré, P., Guasch, M., Moldovan, C., \& Sánchez-Casas, R. (2012). Affective norms for 380 Spanish words belonging to three different semantic categories. Behavior Research Methods, 44(2), 395-403.

[53] Bradley, M., \& Lang, P. (1994). Measuring emotion: The selfassessment manikin and the semantic differential. Journal of Behavior Therapy and Experimental Psychiatry, 25(1), 49-59.

[54] Anooshian, L. J., \& Hertel, P. T. (1994). Emotionality in free recall: Language specificity in bilingual memory. Cognition \& Emotion, 8(6), 503-514.

[55] Ayçiçegi, A., \& Harris, C. (2004). Bilinguals' recall and recognition of emotion words. Cognition \& Emotion, 18(7), 977-987.

[56] Ferré, P., García, T., Fraga, I., Sánchez-Casas, R., \& Molero, M. (2010). Memory for emotional words in bilinguals: Do words have the same emotional intensity in the first and in the second language? Cognition \& Emotion, 24(5), 760-785.

[57] Ferré, P., Sánchez-Casas, R., \& Fraga, I. (2012). Memory for emotional words in the first and the second language: Effects of the encoding task. Bilingualism: Language and Cognition, 16(03), 495-507.

[58] Lee, S. \& Li, D. C. S. (2012). Multilingualism in Greater China and the Chinese language Diaspora, in The Handbook of Bilingualism and Multilingualism. Second Edition (eds T.K. Bhatia \& W. C. Ritchie). John Wiley \& Sons, Ltd., Chichester, UK.

[59] Guide to the Pre-primary Curriculum. (2016) (1st ed., p. 30). Hong Kong. Retrieved from http://www.edb.gov.hk/.../preprimary.../preprimaryguide-net_e

[60] Kroll, J. F., \& Tokowicz, N. (2005). Models of bilingual representation and processing: Looking back and to the future. In J. F. Kroll \& A. M. B. de Groot (Eds.), Handbook of Bilingualism: Psycholinguistic Approaches (pp. 531-553). New York: Oxford University Press.

[61] Degner, J., Doycheva, C., Wentura, D. (2011). It matters how much you talk: On the automaticity of affective connotations of first and second language words. Bilingualism: Language and Cognition, 15(1), 181-189. 